\title{
Neural Network Synthesis of Spin Echo Multiecho Sequences
}

\author{
Stefano Cagnoni, Davide Caramella, Raffaella De Dominicis, and Guido Valli
}

Spin echo multiecho sequences are not frequently used in clinical practice, because they allow the observation of one single slice, imaged at different echo times, for each acquisition. To limit examination time, multislice sequences that include only images derived from one or two echoes are usually acquired. Nevertheless, the strong T2 dependence of multiecho sequences can be used effectively to enhance the contrast between tissues with different $\mathrm{T} 2$ and to gather useful diagnostic information. Artificial neural networks can offer new interesting facilities to the radiologist. In fact, the learning capabilities of neural networks allow them to extract the prototypical behavior of a system from a set of examples. After learning, artificial neural networks can emulate the system behavior even in the presence of new inputs, as far as these are not too different from those included in the training set. A conveniently trained neural network can synthesize a multiecho sequence for each slice of a multislice sequence, requiring only two images for each slice to achieve reliable results. When compared with a true multiecho sequence, the images generated by the network preserve the contrast characteristics of the original ones and have a better signal-to-noise (SNR) ratio. In this paper we report the results achieved by using a neural network to reconstruct synthetic spin echo multiecho images of the brain.

Copyright $\odot 1992$ by W.B. Saunders Company

KEY WORDS: magnetic resonance imaging, spin echo sequences, artificial neural networks, image synthesis.

$\mathbf{M}$ AGNETIC RESONANCE IMAGING (MRI) allows radiologists to obtain different outputs according to preselected acquisition parameters. Before the acquisition of a sequence of images, the operator has to define the orientation of the slicing plane, the number and thickness of slices, the pulse sequence, and related parameters (including repetition time [TR] and echo-delay time [TE] for spin echo sequences) that influence the contrast of the resulting images. ${ }^{1}$

The examination is often performed by a technician who usually follows standard protocols and might not realize when it is necessary to adopt specific acquisition procedures to enhance diagnostically relevant features. When radiologists review the images, there is no longer any possibility of changing the acquisition parameters.

As regards slice orientation, three-dimensional (3D) reformatting can simulate a change of the slicing plane after acquisition. A simple and reliable system for changing and contrast characteristics within the image at the time of review of the examination would be useful.

Theoretically, it would be desirable to reconstruct any type of image (T1-weighted, protondensity weighted, T2-weighted) from a data set obtained with any acquisition parameters. In practice, this "a posteriori" reconstruction is possible only in a limited number of cases. Nevertheless, even within these constraints, such a contrast reformatting could have a significant clinical impact.

For example, when spin echo multislice sequences are acquired, images obtained from the first two echoes could be used to build T2 maps and/or to synthesize a set of images corresponding to different TEs. In the absence of noise, the straightforward fit of a single exponential is sufficient to achieve reliable results. When noise is present, as in an actual acquisition, the reliability of such estimates diminishes. To limit the effects of noise, either more redundant information (a greater number of input images) or a long delay between the acquisition of the two input images can be used. ${ }^{2,3}$ The first remedy has its drawback in requiring that a greater number of images be acquired. With the second one, the values of TE suggested for optimal performances are different from those that are normally adopted, so that either a further sequence must be acquired, or a nonoptimal sequence must be used to perform standard clinical tasks.

In order to solve the aforementioned problems, we have developed and tested a neural network-based system that can reconstruct a

From the Department of Electronic Engineering and the Department of Clinical Physiopathology, University of Florence, Italy; and the Department of Radiology, University of Pisa, Italy.

Supported by grants of MURST (Ministry of University and of Scientific and Technological Research).

Address reprint requests to Stefano Cagnoni, Department of Electronic Engineering, University of Florence, via di S. Marta 3, I-50139 Florence, Italy.

Copyright 11992 by W.B. Saunders Company

0897-1889/92/0502-0001\$03.00/0 
synthetic multiecho sequence from images acquired during a spin echo multislice long-TR/ long-TE pulse sequence. The system allows the examination to be performed with standard methodologies and parameters, thus enabling the radiologist to obtain heavily $\mathrm{T} 2$-weighted images of any of the slices in addition to the ones that are usually available.

A further benefit of the system is an observed enhancement of image quality, with respect to images obtained with true multiecho sequences, resulting from a better signal-to-noise ratio (SNR).

\section{ARTIFICIAL NEURAL NETWORKS}

In 1943 McCulloch and Pitts ${ }^{4}$ devised a mathematical model of the biological neuron that consisted of a summation block into which conveniently weighted input signals were fed. A threshold function was then applied to the sum to determine the neuron output.

Such a model, with minor modifications (the threshold function is usually substituted with a sigmoid function), is still the elementary unit on which artificial neural network architectures are based (Fig 1).

An artificial neural network (ANN) consists of a set of such "neurons," connected together to form a network, according to different paradigms. Usually different layers of processing elements can be distinguished, including neurons characterized by the same distance from the input layer (Fig 2).

The ANNs are characterized by three main properties, which were investigated, and exploited, only long after the work by McCulloch and Pitts: learning, generalization, and abstraction. ${ }^{5}$

The learning process, that can be based on a variety of learning rules, implies the modifica-

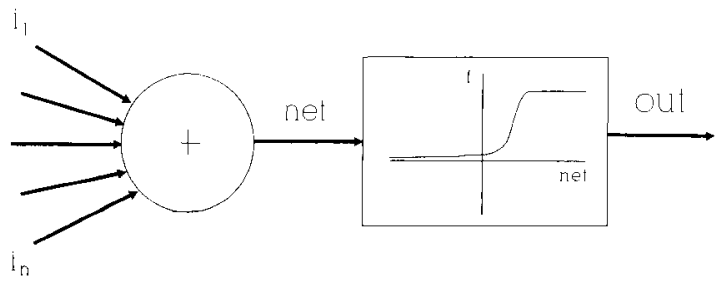

Fig 1. Functional diagram of an artificial neuron: the result of a weighted sum of neuron inputs is fed into a nonlinear block with a sigmoid transfer function, which determines the neuron output.

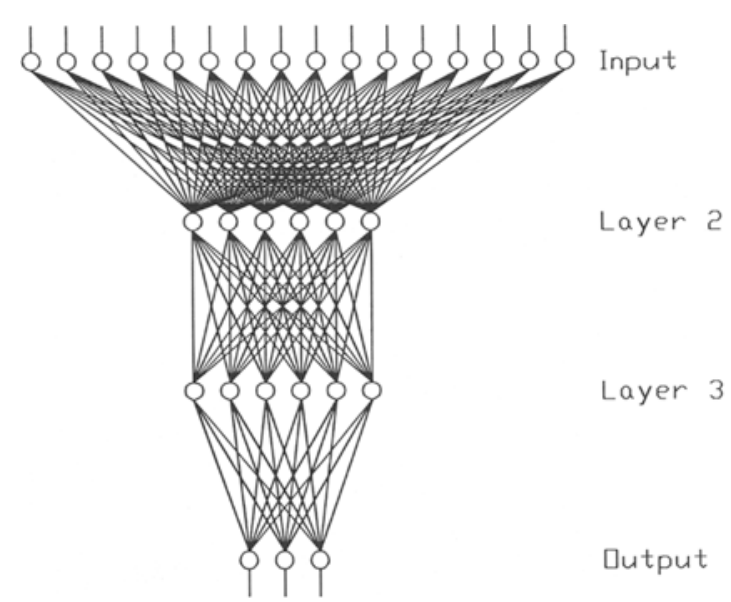

Fig 2. Structure of an artificial feed-forward 4-layer neural network: the outputs of the processing elements of each layer are the inputs for the neurons of a subsequent layer: in this particular case (the network structure used in our work) the network is also full-connected, as all neurons of one layer are connected with all neurons of the following one.

tion of the weights so that the network produces consistent responses to a training set of inputs. The most common learning algorithms do this by minimizing the total squared error (TSE), defined as the sum of the squared differences between the desired outputs and the actual outputs of the network, calculated on the whole training set.

Generalization implies that similar inputs also produce similar outputs. This means that neural networks are insensitive to noise, ie, they can preserve their behavior even in the presence of corrupted or incomplete inputs.

Abstraction refers to the capability of ANNs to extract an underlying prototype from the examples, producing, to some extent, even outputs not previously shown.

These properties have suggested the use of neural networks to solve pattern recognition and classification problems, such as those encountered in character recognition and computer vision. ${ }^{6,7}$ As regards the biomedical field, up to now ANNs have been applied to perform classification or diagnostic tasks through the analysis of monodimensional signals (electrocardiogram [ECG], electroencephalogram [EEG] blood-pressure data, etc).$^{8,9}$

In the field of medical imaging, the solution of problems arising from the analysis of greatly variable 'scenes' suggests that an underlying model be found to describe the physical system 
that has to be analyzed. Nevertheless, such 'a priori' knowledge is often hardly exploitable and, even when the system behavior is described by simple closed-form formulas, traditional approaches often fail when a strong stochastic component (noise) is present.

On the contrary, the development of ANN models of complex systems usually requires not more than the creation of a suitable training set and a few learning sessions (some learning algorithms do not ensure convergence to the global minimum for TSE, so that it is advisable to perform multiple learning sessions).

\section{MATERIALS AND METHODS}

The ANNs used in our study are based on a feed-forward network architecture (signals always propagate towards the output layer), trained with the backpropagation algorithm. ${ }^{10}$ The network is made up of one input, two hidden and one output layers, including 18,6,6 and 3 processing elements, respectively (Fig 2).

The 18 inputs are the gray levels of nine pixels from two corresponding $3 \times 3$ windows in the first and in the second input image. The output values represent the gray level of the central pixel of the window in the resulting long-TE images. The training set used in the learning phase included 4,500 examples, randomly selected from three different coronal spin echo multiecho sequences.

In building the training set a choice had to be made between two approaches: the first suggests that background pixels be included in the training set, and a null desired output be assigned to them; according to the second approach, examples should be taken only from regions of the image, where significant signal is supposed to be found. After trying both approaches, we chose the latter because the former, while correctly setting to zero the background pixels, also produced a suppression of the signal from significant low-intensity areas within the image.

The network was trained by using a network-simulation program we have developed in C language and run on a HP 9000/300 workstation (Hewlett Packard Corp, Palo Alto, CA). Three hundred iterations (about 90 minutes of central processing unit time) were needed for the backpropagation algorithm to converge; nevertheless, the TSE had already reached acceptable values after the first 50 iterations.

Tests were performed by comparing synthetic long-TE images reconstructed from the early echoes of actual multiecho sequences with the corresponding original images.

\section{RESULTS}

Our observations relate to the application of the network to reconstruct synthetic images corresponding to $\mathrm{TE}=120,150,180$ milliseconds, using two images with $\mathrm{TE}=30$ milliseconds and TE $=120$ milliseconds $(\mathrm{TR}=2 \mathrm{sec}$ onds) as inputs.
A comparison between synthetic and original images was performed on a set of coronal multiecho images that had not been included in the training set. A first visual comparison was made by a radiologist, who confirmed that synthetic images are not affected by diagnostically relevant artifacts. In fact, these were found to reproduce the typical behavior of a multiecho sequence, characterized by an increasingly $\mathrm{T} 2$ dependent contrast.

Moreover, the reconstruction of synthetic images yields a better signal-to-noise ratio with respect to the original ones. This suggests that the weights of the neural network represent an optimal model of MR signal. Furthermore, the gray level of each pixel in the output image depends on the gray levels of the corresponding pixel and of the eight surrounding ones in the two input images. This net configuration is likely to produce a smoothing effect. ${ }^{11}$

To quantify the results obtained by the networks, a computer simulated sequence of a phantom was generated (Fig 3). Values for proton density and $\mathrm{T} 2$ that are similar to those of actual brain tissues were given to each region so as to generate a sequence made up of a proton density image and eight echoes, with $\mathrm{TE}$ ranging from 30 to 240 milliseconds. Uniform

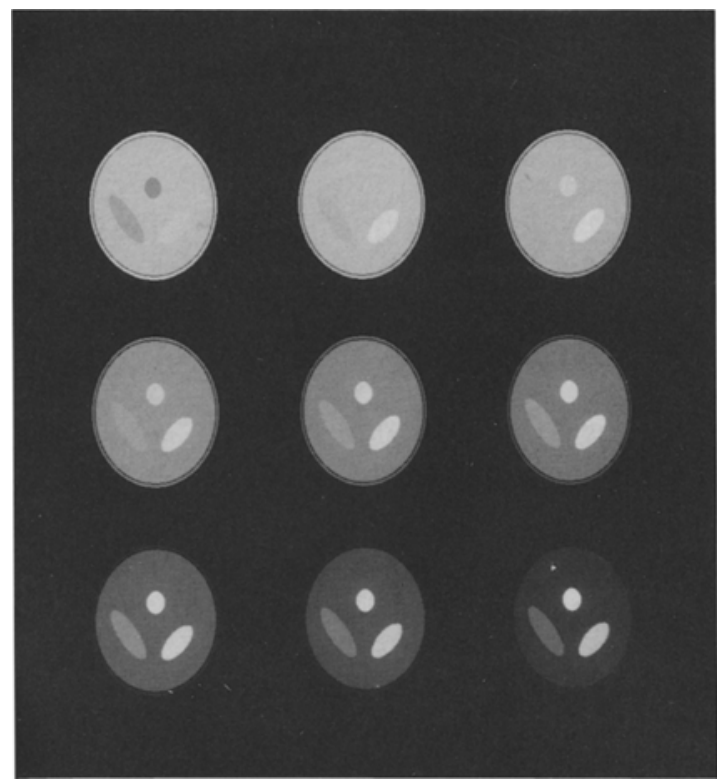

Fig 3. The computer-generated multiecho sequence of a phantom that was used to assess the performances of the network. 
Table 1. Results (TE = 150 milliseconds)

\begin{tabular}{rrrrrrrrr}
\hline & & \multicolumn{3}{c}{ Net } & \multicolumn{3}{c}{ Fit } \\
\cline { 3 - 5 } \cline { 7 - 9 } & Level & $\mu$ & $\sigma$ & $\mu / \sigma$ & $\mu$ & \multicolumn{1}{c}{$\sigma$} & \multicolumn{1}{c}{$\mu / \sigma$} \\
\hline A & 98 & 105.16 & 5.53 & 19.02 & 95.74 & 9.81 & 9.76 \\
B & 129 & 131.44 & 5.71 & 23.02 & 121.62 & 10.42 & 11.67 \\
C & 247 & 229.61 & 6.85 & 33.52 & 222.51 & 11.59 & 19.20 \\
D & 255 & 249.64 & 5.52 & 45.22 & 230.01 & 12.00 & 19.17 \\
\hline
\end{tabular}

NOTE. Results obtained on the sequence shown in Fig. 3 . The neural network and an exponential fit were applied to two images of the phantom corresponding to $T E=\mathbf{3 0}$ milliseconds and $T E=120$ milliseconds, to generate synthetic images corresponding to $\mathrm{TE}=150$ milliseconds (a) and $T E=180$ milliseconds. The "true" gray levels of the four largest regions of the phantom are reported in the first column. The right part of the table reports the mean values $\mu$, the standard deviations $\sigma$, and the signal-to-noise ratios $\mu / \sigma$, characterizing the corresponding regions of the images obtained by the network (left) and by the fit (right).

noise with amplitude of 5 was then added to each image, and gray levels scaled from 0 to 255 . The noise level was chosen in order to obtain images that are close to those obtained by actual spin echo sequences, as regards both mean level and variance of noise.

Two quality factors were chosen to evaluate the performance of the neural network: the first was the difference between the actual gray levels of constant-level regions in the noiseless images and the mean value $\mu$ of pixels in the corresponding regions of the synthetic images; the second was SNR calculated as $\mu / \sigma$ ratio, $\sigma$ being the standard deviation of the signal in the same regions.

A comparison was performed between the synthetic images and those obtained by fitting an exponential curve to the same inputs. The gray levels of the synthetic images are always closer to those of the noisy phantom and SNR is always better (Tables 1 and 2). The resulting images are shown in Fig 4.

The best results were obtained by windowing the actual network output according to two thresholds: the upper threshold is a function of

Table 2. Results (TE $=\mathbf{1 8 0} \mathrm{ms}$ )

\begin{tabular}{|c|c|c|c|c|c|c|c|}
\hline & \multirow[b]{2}{*}{ Level } & \multicolumn{3}{|c|}{ Net } & \multicolumn{3}{|c|}{ Fit } \\
\hline & & $\mu$ & $\sigma$ & $\mu / \sigma$ & $\mu$ & $\sigma$ & $\mu / \sigma$ \\
\hline A & 77 & 82.88 & 4.71 & 17.60 & 74.33 & 9.57 & 7.77 \\
\hline B & 112 & 110.48 & 5.36 & 20.61 & 102.49 & 11.08 & 9.25 \\
\hline C & 220 & 201.29 & 9.69 & 20.77 & 195.24 & 12.99 & 15.03 \\
\hline $\mathrm{D}$ & 255 & 248.14 & 6.95 & 35.70 & 222.22 & 14.50 & 15.32 \\
\hline
\end{tabular}

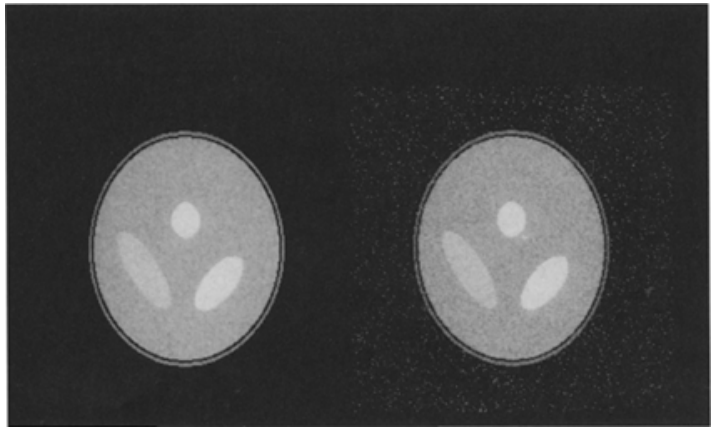

Fig 4. The phantom image, corresponding to $T E=150$ milliseconds, as reconstructed by the network (left), and by performing an exponential fit on the same two input images (right).

the distribution of the highest gray levels in the image, and was chosen so as to bring $1 \%$ of the whole image to saturation (Gray level 255); the lower threshold was calculated by adding half standard deviation of the background noise to its mean value.

The networks were also applied to actual spin echo images. Figure 5 shows the results of the comparison between the gray-level profiles of two corresponding lines of a synthetic image and of the original one.

As shown in Fig 5, the two profiles have the same morphological characteristics; the only distortion introduced by the ANN is represented by minor differences in the gray levels.

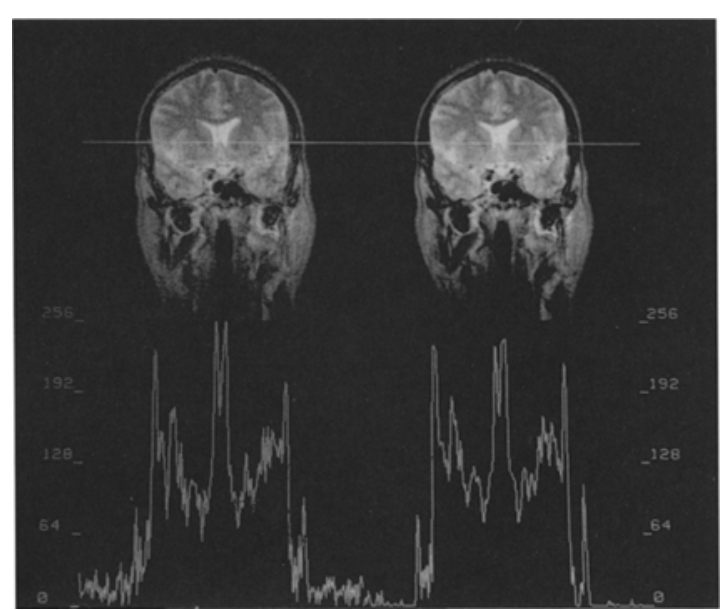

Fig 5. Left: original coronal MR image (TE/TR $=150 / 2000$ milliseconds); right: the corresponding windowed synthetic image. The similarity of the profiles relative to a line passing through the lateral ventricles proves the accuracy of the reconstruction. 

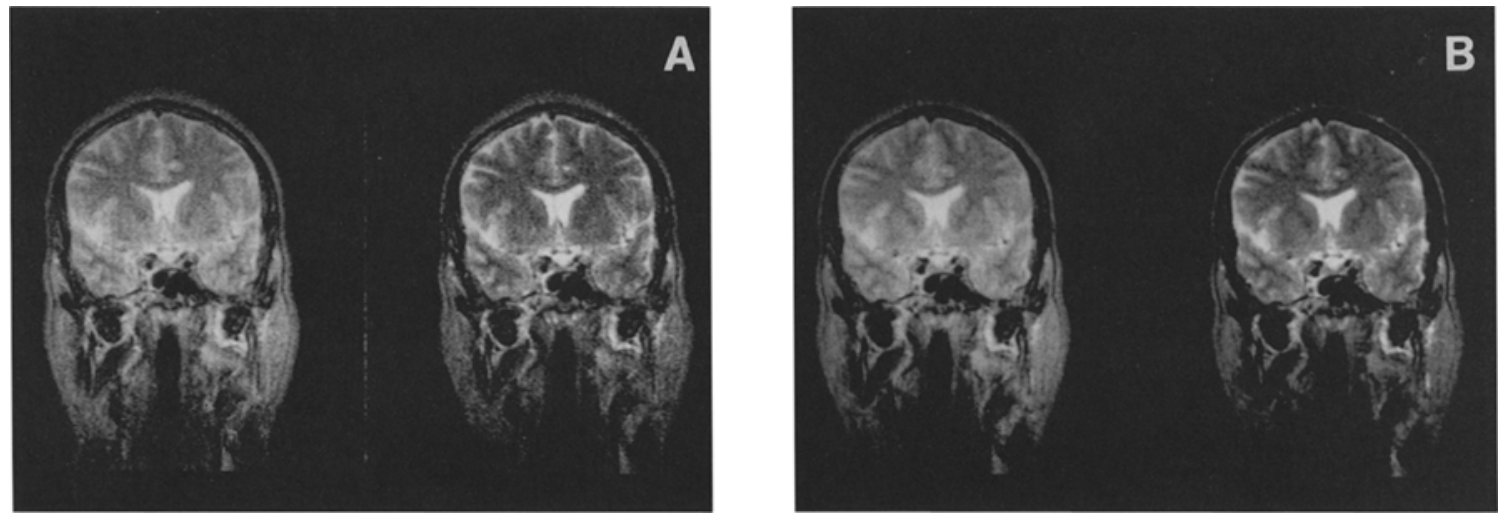

Fig 6. (A) Original coronal MR images (TE $/ T R=150,180 / 2000$ milliseconds); (B) corresponding reconstructed synthetic images, obtained by the network.

The first remark that can be made in comparing the two profiles is that the dynamic range of the network output is compressed with respect to the one of the original image; secondly, the background, apart from being significantly less noisy, is set to a uniform level that is higher than the mean value of noise in the original image. While the latter result is due to the choice to eliminate background pixels from the training set, the compression is physiological for ANNs trained with examples whose desired outputs are not uniformly distributed. In such cases the abstraction property of ANNs causes the distribution of the reconstructed outputs to approximate the distribution of the examples, thus preventing a faithful reproduction of less frequent values.

Such an inaccuracy does not substantially alter the image; in fact, the original aspect of the image can be satisfactorily restored by windowing the images according to the aforementioned procedure.

Figure 6 shows two original images $(\mathrm{TE}=150$ milliseconds, 180 milliseconds) and the corresponding windowed outputs of the networks. To evaluate the abstraction capabilities of the network system, we tested the same network, trained on coronal images, on standard spin echo axial multislice sequences as well. The results of this test are shown in Figure 7. The overall quality of the synthetic axial image is still comparable with that obtained with coronal images, as could be expected.

\section{CONCLUSIONS}

Although applied only on a small set of sequences of brain images, our results seem promising.

A more complex model of the spin echo signal, including parameters to represent noise, etc, might achieve similar or even better results, but its derivation could never be so immediate

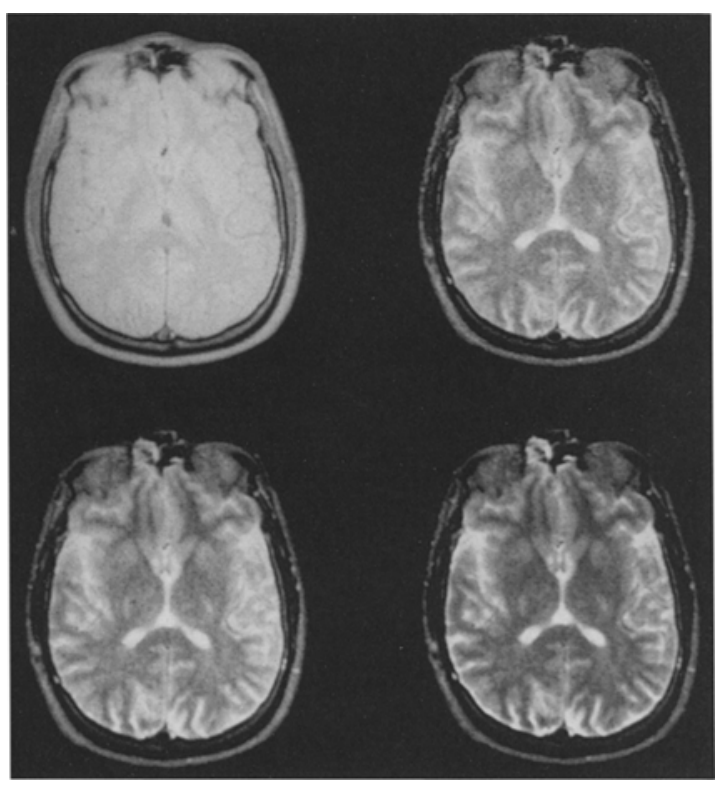

Fig 7. Synthetic images, with TE $/ \mathrm{TR}=150,180 / 2000 \mathrm{milli}-$ seconds (bottom row) obtained by applying a network, trained by coronal images, on a standard axial sequence with TE/TR = 30,120/2000 milliseconds (top row). The good results confirm the abstraction capabilities of neural networks. 
and generally applicable as with the learning procedures typical of ANNs.

Indeed, the use of a neural network approach to MR image synthesis showed the double advantage of retrospectively obtaining long-TE spin echo images from a standard two-echo multislice sequence and of improving image quality, thanks to the better SNR of synthetic images in comparison with those obtained by actually performing a multiecho acquisition.

\section{REFERENCES}

1. Brant-Zawandzki M, Norman D: Magnetic resonance imaging of the central nervous system. New York, NY, Raven, 1987

2. Darwin RH, Drayer BP, Riederer SJ, et al: T2 estimates in healthy and diseased brain tissue: a comparison using various MR pulse sequences. Radiology 160:375-381, 1986

3. McFall JR, Riederer SJ, Wang ZW: An analysis of noise propagation in computed T2, pseudodensity, and synthetic spin-echo images. Med Phys 13:285-292, 1986

4. McCulloch WS, Pitts W: A logical calculus of the ideas immanent in nervous activity. Bulletin Math Biophys 5:115133,1943

5. Wassermann PD: Neural computing. Theory and practice. New York, NY, Von Nostrand Reinhold, 1989

6. Fukushima K, Wake N: Handwritten alphanumeric character recognition by the Neocognitron. IEEE Trans Neural Networks, 2:355-365, 1991

7. Bilbro GL, White M, Snyder W: Image Segmentation with Neurocomputers, in Eckmiller R, vd Malsburg C (eds): Neural Computers, Berlin, FRG, Springer-Verlag, 1987

8. Special issue on Applications of Neural Networks. IEEE Eng Med Biol Soc 9:3, 1990

9. Poli R, Cagnoni S, Coppini G, et al: A neural network expert system for diagnosing and treating hypertension. Computer 24:64-71, 1991

10. Rumelhart DE, Hinton GE, Williams RJ: Learning internal representations by error propagation, in Rumelhart DE, McClelland JL (eds): Parallel distributed processing. Explorations in the microstructure of cognition (vol 1). Cambridge, MA, MIT Press, 1986

11. Rosenfeld A, Kak A: Digital picture processing. Orlando, FL, Academic, 1981 